6- Para executar corretamente a técnica através do uso de vídeo instrucional, é necessário passar o mesmo quantas vezes?
() 1 vez
( ) 2 vezes
( ) 3 vezes
( ) 4 vezes
( ) mais de 5 vezes

7. Sugestões:

\title{
PREVALÊNCIA DE DESNUTRIÇÃO PROTÉICO-ENERGÉTICA E EVOLUÇÃO NUTRICIONAL DE PACIENTES INTERNADOS EM ENFERMARIA DE CLÍNICA MÉDICA
}

\author{
JANE BANDEIRA DICHI 3 \\ MÔNICA DO VALE ROCHELLE ${ }^{2}$ \\ EDNAMAR APARECIDA SENNE ${ }^{2}$ \\ MARCOS CESAR BARROS DE ALMEIDA CAMARGO' \\ ISAIAS DICHI ${ }^{3}$
}

DICHI, Jane Bandeira; ROCHELLE, Monica do Vale; SENNE, Ednamar Aparecida; CAMARGO, Marcos Cesar Barros de Almeida; $\mathrm{DICHI}$, Isaias. Prevalência de desnutrição protéico-energética e evolução nutricional de pacientes internados em Enfermaria de Clínica Médica. Semina: Ci. Biol./Saúde, Londrina, v. 16, n. 2, p. 219-223, jun. 1995.

RESUMO: A avaliaçăo do estado nutricional (antropometria e parâmetros laboratoriais) foi realizada em 92 pacientes $(61 \mathrm{M}$ e 31F) de $47+19$ anos, internados na Enfermaria de Clínica Médica deste Hospital durante um ano. Estes pacientes eram portadores, em sua maioria, de patologias gastrointestinais (19\%), neoplasias (18\%), cardiovasculares (12\%) e hepatopatia crônica (11\%). Quando comparados com valores de referência, verificou-se, pela associação dos resultados dos indicadores nutricionais, que $60 \%$ dos pacientes encontravam-se desnutridos ou com risco nutricional na internaçăo, sendo este índice de $72 \%$ na alta hospitalar. O tempo médio de internação foi de $15+8$ dias. Dentre as doenças prevalentes, verificou-se maior incidência de desnutrição e risco nutricional nos pacientes portadores de úlcera péptica (100\%) e neoplasias $(70,6 \%)$. Com relação aos indicadores nutricionais individualiza. dos, verificou-se que a maioria dos pacientes apresentava um maior comprometimento dos estoques de proteina somática do que dos estoques de gordura. Assim, pode-se concluir que a prevalência de desnutrição, entre estes pacientes, é alta, não diminuiu com a internação e que, apesar da melhora clínica, năo houve melhora nutricional concomitante.

PALAVRAS CHAVE: Avaliaçăo do estado nutricional; desnutrição hospitalar.

\section{INTRODUGÃO}

Na década de 70, BISTRIAN et al. $(1974,1976)$ constataram um percentual elevado $(50 \%)$ e pacientes já desnutridos no momento da internação hospitalar ou que s desnutriam no decorrer da mesma, em enfermarias de Clínica Médica e Cirurgia Geral. Esta desnutrição intra-hospitalar pode ocorrer devido a vários fatores, como jejum para exames, dietas restritas em proteínas e/ou sódio, infecções, estresse cirúrgico, etc. (BLACKBURN
\& HARVEY, 1982; McCULLOUGH et al., 1989). A partir daí, estes dados foram confirmados em um grande número de publicações na literatura (DICHI et al., 1993 DICHI et al., 1991C; MARCHINI et al., 1986; MULLEN et al., 1979; WEINSIER et al., 1979). A importância deste achado se deve ao fato qu a desnutrição protéicoenergética não apenas aumenta o tempo de internação dos pacientes, como também aumenta a morbidad e mortalidad dos mesmos (ANDERSON et al., 1984; BISTRIAN et al., 1975; BLACKBURN \& HARVEY, 1982;

1 - Professor da disciplina de Semiologia e Clinica Médica - Departamento de Clínica Médica - CCS - Universidade Estadual de Londrina

Londrina, PR, Brasil, Caixa Postal 6001, CEP 86051-970.

2 - Nutricionistas estagiárias da disciplina de Semiologia e Clínica Médica.

3 - A quem toda correspondência deve ser endereçada.

Semina Ci. Biol./Saíde, v. 16, n. 2, p. 214-219 
BLACKBURN \& THORNTON, 1979; MURRAY et al., 1988; WEINSIER et al., 1979).

Assim, a avaliação do estado nutricional (AEN) mediante inquérito alimentar, antropometria e exames laboratoriais, deve-se constituir no primeiro passo para uma intervenção nutricional eficaz. Em realidade, através da A.E.N. criteriosa, não só diagnosticamos desnutrição como também podemos detectar pacientes com risco nutricional, sendo que este último diagnóstico passaria despercebido se fosse realizado apenas o exame clínico convencional.

Apesar disso, ainda são poucos os grupos de profissionais existentes em nosso meio voltados para a detecção de desnutrição em pacientes hospitalizados em enfermarias de Clínica Médica (MARCHINI et al., 1986).

Assim, este trabalho tem como objetivo avaliar o estado nutricional de pacientes hospitalizados, e acompanhar a sua evolução nutricional durante a internação, destacando o envolvimento nutricional encontrado nas doenças de maior prevalência.

\section{PACIENTES E MÉTODOS}

Foram analisados 92 pacientes internados na Enfermaria de Clínica Médica do Hospital Regional do Norte do Paraná, no período de Abril/1992 a Março/1993. Fodos os pacientes receberam durante a internação a dieta geral hospitalar. O diagnóstico clínico dos pacientes avaliados encontra-se na Tabela 1. A média de idade dos pacientes foi de $47+19$ anos (variando de 13 a 118 anos); 61 eram do sexo masculino e 31 do sexo feminino. O período de internação foi de $15+8$ dias.

$A$ avaliação nutricional foi realizada, semanalmente, meidante antropometria e exames laboratoriais. $\mathrm{Na}$ antropometria foram verificados os valores de peso $(P) e$ altura (h) (em balança antropométrica Filizola), prega cutânea tricipital (PCT) (com calipímetro CESCORF Porto Alegre - RS) e circunferência braquial (CB) (com fita de celulose, milimetrada, inextensível) no ponto médio do braço entre o acrômio e o olécrano (BLACKBURN \& THORNTON, 1979). A partir destes dados foram calculados os indices derivados: índice de Quetelet (IQ: P/h2) e circunferência muscular do braço (CMB) (BLACKBURN \& THORNTON, 1979).

Em amostras colhidas pela manhã, após periodo de repouso e jejum de $8-12$ horas, foram realizados os seguintes parâmetros sanguineos: albumina (método do verde de bromocresol - Autoanalizer, RA - 1000, Technicon) e contagem de linfócitos totais.

Os resultados obtidos foram comparados com os padrões de referência, de acordo com a distribuição no percentil obtido na antropometria (CRONCK \& ROCHE, 1982; FRISANCHO, 1981) e nos parâmetros laboratoriais (MATHIAS et al., 1986).

Conforme recomendação da literatura (BLACKBURN \& HARVEY, 1982; BLACKBURN \& THORNTON, 1979), os pacientes foram classificados como desnutridos quando apresentavam 3 indicadores nutricionais abaixo do $5^{\circ}$. percentil, e quando apresentavam 2 indicadores nutricionais abaixo do $5^{\circ}$. percentil eram considerados como de risco nutricional. 0 sobrepeso e a obesidade foram caracterizados pelo indice de Quetelet, acima de 27 a $30 \mathrm{~kg} / \mathrm{m}^{3}$, respectivamente (BRAY, 1989).

$A$ avaliação nutricional nos momentos da intemação e da alta hospitalar foi obtida em 33 pacientes.

\section{RESULTADOS}

$A$ avaliação do estado nutricional dos pacientes no momento da internação encontra-se na Tabela 2. Verifica-se que $61 \%$ dos pacientes apresentavam-se desnutridos ou com risco nutricional. Na Tabela 3 , encontra-se a avaliação nutricional dos $33(36 \%)$ pacientes em que foram realizadas mais de uma avaliação nutricional. Verificou-se um aumento de $12 \%$ na incidência de desnutrição ou risco nutricional na alta destes pacientes.

Na Tabela 4, verificou-se uma incidência elevada de desnutrição ou risco nutricional (100\%) nos pacientes portadores de úlcera péptica.

Dentre as patologias mais frequentemente encontradas (Tabela 5), verificou-se um comprometimento maior da proteína somática (CMB) nos pacientes portadores de neoplasias, ICC e úlcera péptica, enquanto nos hepatopatas crônicos o comprometimento dos estoques de gordura (PCT) foi maior.

\section{DISCUSSÃO}

Verificou-se no presente trabalho uma incidência de desnutrição protéico-energética (DPE) e risco nutricional em pacientes hospitalizados em Enfermaria de Clínica Médica superior a 50\%, resultado comparável ao de outros trabalhos (BISTRIAN et al., 1976; KAMATH et al., 1986; WEINSIER et al. 1979). Entretanto, apesar da internação, os 33 pacientes que tiveram mais de uma avaliação, apresentaram mediante a associação dos indicadores nutricionais um aumento de $12 \%$ nesta incidência quando da alta hospitalar (Tabela 3). Este achado, encontrado também em outros trabalhos (DICHI et al., 1991c; WEINSIER et al., 1979), mostra que a alta dos pacientes ainda se baseia quase exclusivamente em critérios clinicos, que, praticamente, não levam em conta o estado nutricional dos pacientes (DICHI et al., 1991a; PAPINI-BERTO et al., 1992).

Apesar de, classicamente, apenas a úlcera gástrica estar associada à perda de peso (MCGUIGAN, 1991), os pacientes portadores de úlcera duodenal, quando internam, o fazem devido a alguma complicação, e, no nosso caso, isto se deveu à hemorragia digestiva e à estenose do piloro, o que pode justificar a frequência de $100 \%$ de desnutrição ou risco nutricional encontrada nestes pacientes. Esta incidência foi, inclusive, superior à encontrada nos pacientes portadores de neoplasias que também apresentaram uma incidência alta de DPE e/ou risco nutricional (70\%). Cabe ressaltar que, em sua 
grande maioria, estes pacientes apresentavam neoplasias localizadas no trato gastrointestinal. SabeSE ofue diagnóstico secundário mais frequente nos pacientes portatores de meoplasia é a desnutrição, conseguenche dire? maligniolades (HARVEY of al., 1979). Além disso, esies pacientes preseniam nos casos avançados um annento do gasto enengérico e do catabolismo protéico GORELEN \&U, 1990).

Tamberm, mais de $50 \%$ dos pacientes portadores

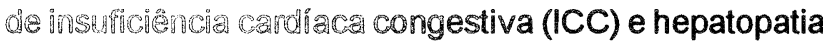
Crônica aresentaram DPE e/ou risco nutricional. $\mathrm{Na}$ ICC. anorexis ensiderada uma das causas princi-

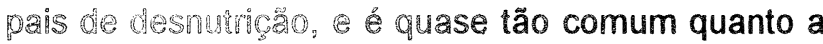
dispreig elou edema. Entre os fatores causais de redu-

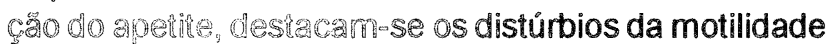
gastrointestinal. dor a compressão abdominal decorrenles da heparonegaha, dispnéia e hipoxemia, efeitos colaterais ole nedicamentos, adoção de dietas restritas e hipennetabolismo (VELLOSO et al., 1992). Apesar de Serem inumeros os farores responsáveis pela DPE na ICG, a requência de desnutriç̃o e risco nutricional encontraca (56\%) 10 m minto elevada quando comparada a Ontros trabalhos (BOLLET \& ONENS, 1973; VELLOSO (at al. 1992). Por outro lado, a frequência de desnutri-

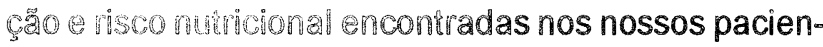
les com hepatoparia crônica é comumente vista na lite-

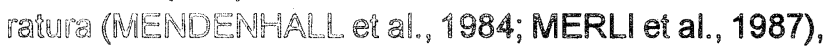
mesmo en pacientes com cirrose hepática em sua fase inicisl (OICHil of ar. 1992; MERLI et al., 1987). Na hepontopatia crônics, a desnutriçào é multifatorial, e piora com o agravamento da função hepática (DICHI et al. 1991b) cabendo salientar as causas de desnutrição intrahospitalar deviolo æo jejum prolongado para realização de exames, sangranento digestivo, alterações no nivel consciencive dietas especiais, hipossódicas para pacientes porracores de ascite (DICHI et al., 1993) e hopoproiéicas para pacientes portadores de encefalopatia hepática (OLCHI \& BURIN, 1994). Evidentemente, o jejum prolongado e repeido para realização de exames é Causa de DPE em oulouer paciente hospitalizado, mas aspeciannente nos pacientes portadores de patologias do tubo digestivo, onde, comumente, a prevalência de

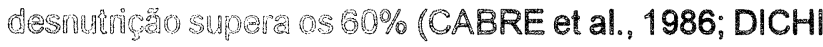

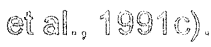

Coberind destacar que, dentre os 33 pacientes que hiverm mais de una avaliação, oito (24\%) portadores de $10 \mathrm{C}$ ou hepatopatia crônica apresentavam edema

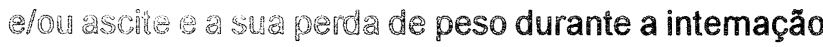
poderiva ter tontuibuido para uma classificação errônea de piora do estaco nutricional, no momento da alta (DICHI (BUSNA, 4992). Entretanto, fato inverso ocorre com a alburnina nestes pacientes. No momento da intemação, OS niveis: séricos de aloumina estão sujeitos a uma in.

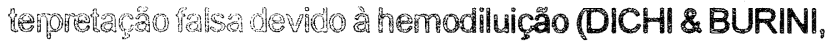

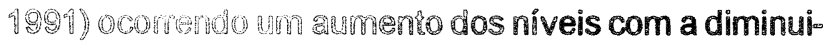

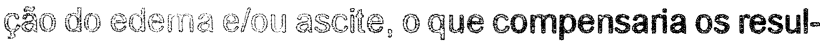
tados obnotos com 0 peso as indices dele derivados
(IQ). Estes dados ajudam a enender a innontancia da utilizaça de mais de um indicador nutricional para a elaboraço de uma classificaço nutricional (DELHEY et al., 19898 .

Com relaça mos indicadores nutricionas individualizados das patologias mais encontraces, omaior comprometimento da proteina Somåtica en relag:a a as estoques de gordura encontrados mos pacientes portadores de neoplasias, ICC e úlcer pépricar demonstra 0 nivel acentuado de graviodade da doenga onanoo os pa-

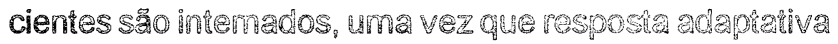

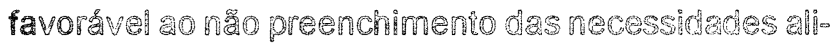
mentares seri⿵ mais às custas dos estoques de gordu ra (CAHLL, 1970), o que näo ocoreu no presente urabztho. Cabe ainda desiacar que muiros pacienies foram internados devio a inercontencias aguors destas doenças crônicas, como inreç̧⿻, hemomagia digesinva, etc. que fambém poderiam justîncar un maior compro. metimento da protema somática. Alem disso, os pacientes com neoplasias, na presenca de jejun. são me nos capazes de conservar a massa magra corporal de vido a sua incapacidade de diminuir alicogenese a pariv oos estoques proteicos (BRENAMN, 1977 ).

De modo geral, houve tamben un grande numero de pacientes que apresentavam niveis sericos de abbmina e contagen de lintócítos abako do 5 - percentil (Tabela 5). A queda da albumina poderia ser explicada por una dininuiça de sintese na hepałopaî crônica $e$ neoplasias, por hemodinicão nos prientes con ICC ou com, hepatopatia crônica poradores de edema elou ascite e por um comprometimento do estado nutricional em qualoner das patologias referidas (DICHI B BURIN.

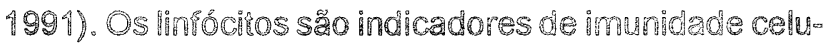

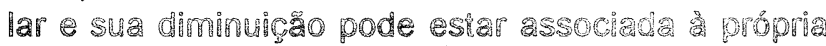
doenç nos casos de neoplasia neparopania crônica

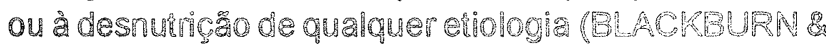
MARVEY, 1982).

\section{CONCLUSEES}

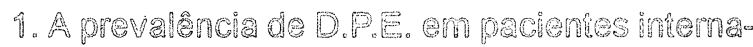

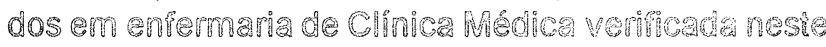
trabaho tura.

2. Amehora chica dos pacientes niga hoi acom-

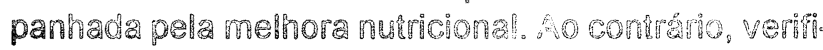
cou-se um anmento no munero de pacientes osmuth. dos ou com risco murricional durante a memaça.

3. Dentre as patologis mais prequenes. verimcouse que a maior inciąencia de desnutriga ocorreu nos pacientes portadores de úlcera pepica.

4. Pontanto, toma-se mecessano 0 dignosico

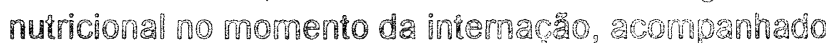
de atenço especial para as parologias cansandors de

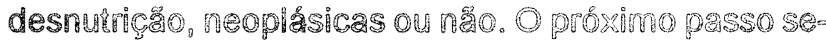

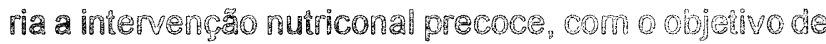

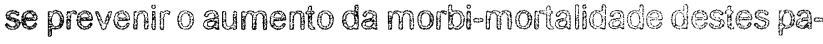
cientes durante a inemaça hospiralar. 
TABELA 1: DIAGNÓSTICOS CLÍNICOS DOS PACIENTES INTERNADOS.

\begin{tabular}{lrr}
\hline DIAGNÓSTICO CLÍNICO & $\mathbf{N}^{*}$ & $\mathbf{( \% )}$ \\
\hline DOENÇAS GASTROINTESTINAIS & 18 & $(19)$ \\
NEOPLASIAS & 17 & $(18)$ \\
DOENÇAS CARDIOVASCULARES & 12 & $(12)$ \\
HEPATOPATIA CRÔNICA & 11 & $(11)$ \\
DOENÇAS INFECCIOSAS & 6 & $(6)$ \\
LITIASE BILIAR & 6 & $(6)$ \\
INTOXICAÇÕES EXÓGENAS & 6 & $(6)$ \\
ANEMIAS & 5 & $(5)$ \\
DIABETES MELLITUS & 4 & $(4)$ \\
OUTROS & 12 & $(12)$ \\
\hline TOTAL & & \\
\hline
\end{tabular}

* Número de vezes que ocorreu o referido diagnóstico, incluindo os casos em que houve mais de um diagnóstico por paciente.

TABELA 2: AVALIAÇÃO DO ESTADO NUTRICIONAL DOS PACIENTES NO MOMENTO DA INTERNAÇĀO $(n=92)$.

DESNUTRIÇÃO 42,4

RISCO NUTRICIONAL 18,5

EUTRÓFICO

SOBREPESO

OBESO

$n=$ número de pacientes.
TABELA 3: AVALIAÇĀO DO ESTADO NUTRICIONAL DOS PACIENTES COM MAIS DE UMA AVALIAÇÃO $(n=33)$.

\begin{tabular}{lrr}
\hline & ENTRADA & SAIDA \\
\hline DESNUTRIDO & $39 \%$ & $42 \%$ \\
RISCO NUTRICIONAL & 21 & $30 \%$ \\
EUTRÓFICO & $33 \%$ & $21 \%$ \\
SOBREPESO & $0 \%$ & $0 \%$ \\
OBESO & 6 & 6
\end{tabular}

$n=$ número de pacientes.

TABELA 4: FREQUENNCIA (\%) DE DESNUTRIÇÃO DAS PATOLOGIAS MAIS ENCONTRADAS.

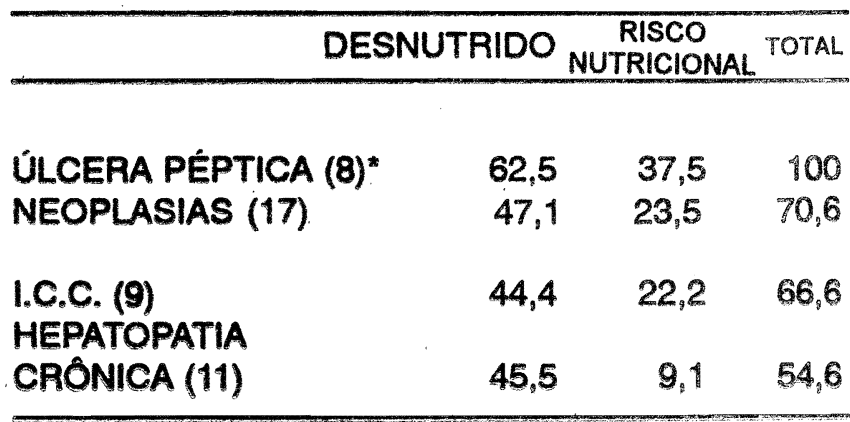

* = número de pacientes

TABELA 5: FREQUENCIA (\%) DOS INDICADORES NUTRICIONAIS <P5 NAS PATOLOGIAS MAIS ENCONTRADAS.

\begin{tabular}{lccccc}
\hline & IO & PCT & CMB & $\begin{array}{c}\text { ALB, } \\
\text { SERICA }\end{array}$ \\
\hline NEOPLASIAS (17) & & \multicolumn{5}{c}{52,9} \\
HEPATOPATIA & 41,2 & 35,3 & 52,9 & 47,1 & 58,8 \\
CRÓNICA (11) & 18,2 & 54,5 & 45,5 & 45,5 & 45,5 \\
I.C.C. (9) & 33,3 & 22,2 & 44,4 & 33,3 & 33,3 \\
ÚLCERA PEPTICA (8) & $\mathbf{7 5 , 0}$ & 50,0 & $\mathbf{8 7 , 5}$ & 37,5 & 50,0 \\
\hline
\end{tabular}

- número de pacientes

1Q: indice de Quetelet; PCT: prega cutanea tricipital;

CMB: circunferencla muscular do braço; Alb: albumina; linf: linfócitos
254.124/91.

Ao prof. Álvaro Jabur, Diretor-Superintendente do H.U.R.N.P., pelo apoio conferido para a realização deste trabalho.
Este projeto foi realizado com recursos obtidos junto à CPG - Diretoria de Pesquisa da UEL, processo $n^{\circ}$.

\section{AGRADECIMENTOS}

DICHI, Jane Bandeira; ROCHELLE, Monica do Vale; SENNE, Ednamar Aparecida; CAMARGO, Marcos Cesar Barros de Almeida; DICHI, Isaias. Prevalence of protein-energy malnutrition and nutritional evolution in hospitalized patients. Semina: Ci. Biol./Saúde, v. 16, n. 2, p. 219-223, Jun. 1995.

ABSTRACT: The nutritional assessement by anthropometric and blood components was undertaken in 92 adult patients, 61 males and 31 females, suffering from gastrintestinal (19\%), cancer (18\%), cardiovascular (12\%), chronic hepatic (11\%) diseases and others. The nutritional evaluation was repeated at the entry and at the discharge of the hospitalization which lasted 15 days. The undernutrition at the admission was of $60 \%$ and at the discharge of $72 \%$. Undernutrition was higher in patients with peptic ulcer (100\%) and cancer $(70,6 \%)$. Protein somatic involvement was higher than the fat stores. Thus, the prevalence of undemutrition is high in Internal Medicine wards and despite clinical improvement there was no concomitant improvement of the nutritional status.

KEYWORDS: Nutritional assessement; hospital malnutrition. 


\section{REFERÊNCIAS BIBUOGRÁFICAS}

ANDERSON, S.F.; MOXNESS, K.; MEISTER, J.; BURRIT, M.F. The sensitivity and specificity of nutrition-related variables in relationship to the duration of hospital stay and the rate of complications. Mayo Clin. Proc., V.59, $977-83,1984$.

BHSTRIAN, B.R.; BLACKBURN, G.L.; HALLOWELL, E., HEDDLE, Rrotein status of general surgical patients. JAMA v. 230, 10.858-60, 1974.

BISTRIAN, 8.R.; BLACKBURN, G.L.; SHERMAN, M., SCRINSHAW, N.S. Therapeutic index of nutritional depletion in hospitalized patients. Surg. Gynecol. Obst. V. 14. 1. 5. 512-6, 1975 .

BISTRRAN, B.R。; BLACKBURN, G.L.; VITALE, J.; COCHRAN, D.; NAYLOR, $\rfloor$ Prevalence of malnutrition in general medical patients. JAMA, v. 235, p. 1567-70, 1976.

BLACKBURN, G.L.; HARVEY, K.B. Nutritional assessement as a routine in clinical medicine. Postgrad. med., v. 71, p. 46-63, 1982 .

BLACKBURN, G.L.; THORNTON, P.A. Nutritional assessement of the hospitalized patients. Med. Clin North. Am., v. 63, p. 1103-15, 1979.

BOLLET, A.J.; OWENS, S. Evaluation of nutritional status of selected hospitalized patients. Am. J. Clin. Nutr., v. 26, p. $931-8,4973$

BRAY, G.A. Classificacão e avaliação das obesidades. Clin. Med. Am. Norte, v. 1, p. 189-216, 1989.

BRENNAN, M.F. Uncomplicated starvation vs. cancer cachexia. Cancer res., v. 37, p. 2359-64, 1977.

CABRÉ, E。: MONTSERRAT, A.; VILAR, L.L.; ABAD, A.; GASSUL, M.A. Prevalencia da malnutrición energético. protéica (M.E.P.) en pacientes gastroenterológicos. Rev. Esp. Enf. Ap. Digest., v. 70, p. 241-6, 1986.

CAHML, G.F. Starvation in man. N. Engl. J. Med., v. 282, D. 668 75, 1970 .

CRONK, C.E.; ROCHE, A.F. Race and sex-specific reference data for triceps and subscapular skinfolds and weight/ stature. Am. J. Clin. Nutr., v. 35, p. 347-54, 1982.

DELHEY, D.M.; ANDERSON, E.J.; LARAMEE, S.H. Implications of mainutrition and diagnosis-related groups (DRGs). J. Am. Diet. Assoc., p. 1448-51, 1989.

DICHI, I.; BURINI, R.C. Metabolismo e regulação dos níveis plasmáticos de albumina. Cadernos de Nutrição, v. 3. p. $99.127,1991$.

DICHI, I. PAPINI-BERTO, S.J.; DICHI, J.B.; DILELLO, A.; VICTÓRIA, C.R.; BURINI, R.C. Implicações nutricionais no tratamento e recuperação de pacientes adultos com diarréia crônica. Arg. Gastroenterol., v. 28, p. 86-92, 19912.

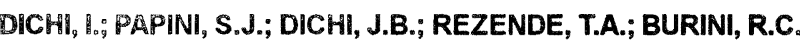
Estado nutricional de pacientes portadores de cirrose hepática. Relação entre a gravidade da doença com a piora nutricional avaliada por indicadores antropométricos e bioquímicos. Rev. Bras. Nutr. Clín. Yo 6. 20.33, 1991b

DICHH, A. B.; BURINI, R.C. Avaliação do metabolismo protéico em humanos. Cadernos de Nutrição, v. 5 , p. $18.35,1992$.

DICHI, 』.: BURINI, R.C. Fundamentos metabólicos da diecoterapia (nitrogenada) de pacientes cirróticos com encefefalopatia aguda on crónica. Rev. Metab. \& Nutrição, V. $1,0 \% 15.20,1994$

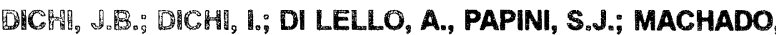
M.M.:BURINI, R.C. Consequencias nutricionais da alefoterapia restrita em sódio $(50 \mathrm{mEq}) \mathrm{em}$ pacientes cirrótícos com ascite. Rev. Bras. Nutr. Clin., v. 8, p. 11-13, 4993.

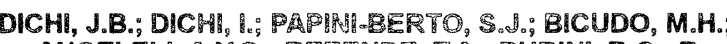
ANGELELI, A. Y.O. REZREDE, T.A.; BURINI, R.C.Resposta metabólic de pacienêses cincóticos (Child A) ao

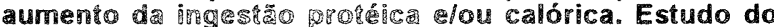
balango nürogende. Arq. Gastroenterol., v. 29, p. $128-136,1932$.

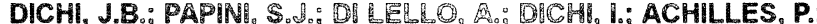

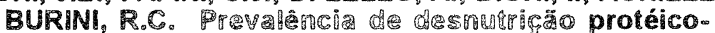

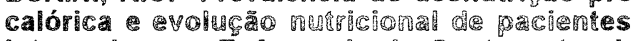
internados Em Erem

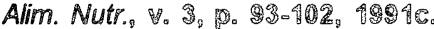

DREIZEN, S.; MCCREDIE, R.B.; MEATUNG, M.H.ANOERSSON, B.S. Nutritional deficiencies in patients receiving cancer chemotheraphy. Postgrad Med., $v_{0} y^{2}, 0.163-70$, 1990.

FRISANCHO, A. R. New nomms of upper limb fấ muscle areas for assessement of mutritional statrs. Am. J. Clin. Nutr., *. 39, p. 20.45, 1981.

HARVEY, K.B.;BOTHE, A。; BLACRBURE, G.L. Mratritional

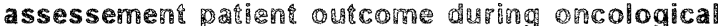
therapy. Cancer, $v, 43,0,2065,927 \%$

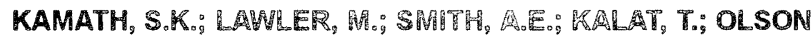
R. Hospital Malnutrition: A 33 -hospital screening study. J. Am. Diế. Assoc., $\vee .60,203-1986$.

MARCHIN!, B.S.; ANSELMO, M.A.C.; BURIN, R.C.Evolução do estado mutritional de pacientes internados em

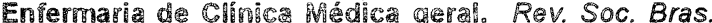

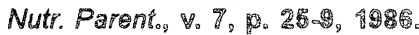

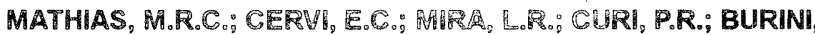
R.C. Estabelecimento dos foind normalidade de variáveis hematológicas bioqumicas de soro de

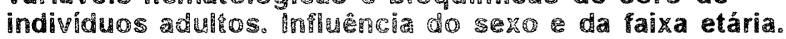
Rev. Bras. Pat. Clin., v. 26. 10. 106 12986.

MCCULLOUGH, M.

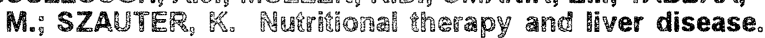

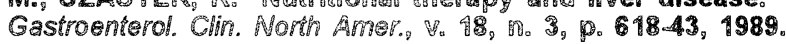

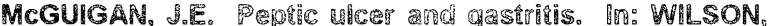

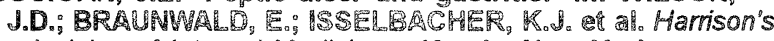
principles of internal Viedicine. 12 ed. Rew Yor:

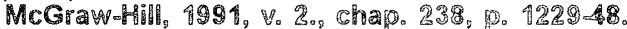

MENDENHALL, CLOANDERSON, S.9 MESESER, R.E.;

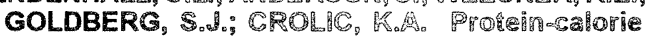

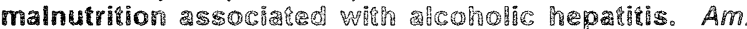
J. Med., Y. 76, 10. 21

MERLI, N:: ROMITI, A.: RIGGO, O. CAPOCACGI, L. Optimal nutritional indeses in chronic liver disease. J. Parenter. Enteral. Nutr. $\%$ 14, 19. $130 S$ AS, 1987.

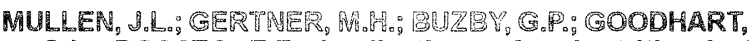

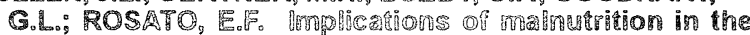

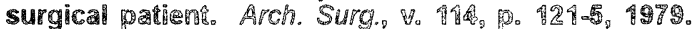

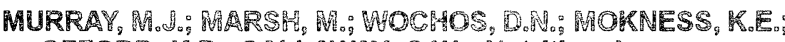

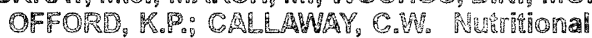

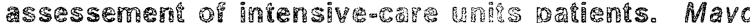

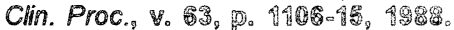

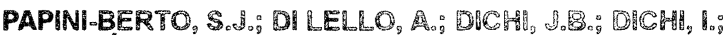

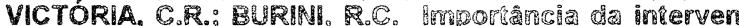
cão chinca mutricion an majentes gastroenterológicos: rebato desos. R. Nutr. PUCCAMP, V. 5. 28.42, 4992.

VELLOSO, LG. CSEMGER C.MLL; BARRETO,

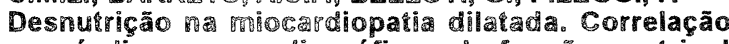

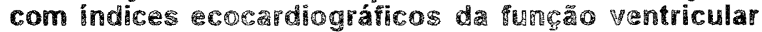

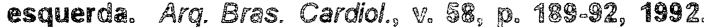

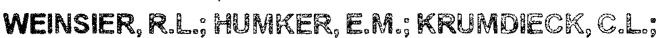
BUTTERWORTH, C.E. HOSpital malrertriajom: a evolution

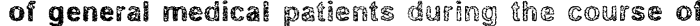
hospitalization. Am. J. Cin. Nest., v.32, P.

Semina Ci. Biol./Saúde, v. 16, n. 2, p. 219-223 